\title{
Timing of developmental reduction in epithelial glutathione redox potential is associated with increased epithelial proliferation in the immature murine intestine
}

\author{
Graham K. Reid ${ }^{1}$, Andrew J. Berardinelli ${ }^{1}$, Laurie Ray ${ }^{1}$, Arena R. Jackson ${ }^{1}$, Andrew S. Neish ${ }^{2}$, Jason M. Hansen ${ }^{3}$ and \\ Patricia W. Denning ${ }^{1}$
}

BACKGROUND: The intracellular redox potential of the glutathione (GSH)/glutathione disulfide (GSSG) couple regulates cellular processes. In vitro studies indicate that a reduced GSH/GSSG redox potential favors proliferation, whereas a more oxidized redox potential favors differentiation. Intestinal growth depends upon an appropriate balance between the two. However, how the ontogeny of intestinal epithelial cellular (IEC) GSH/GSSG redox regulates these processes in the developing intestine has not been fully characterized in vivo.

METHODS: Ontogeny of intestinal GSH redox potential and growth were measured in neonatal mice.

RESULTS: We show that IEC GSH/GSSG redox potential becomes increasingly reduced (primarily driven by increased GSH concentration) over the first 3 weeks of life. Increased intracellular GSH has been shown to drive proliferation through increased poly-ADP-ribose polymerase (PARP) activity. We show that increasing IEC poly-ADP-ribose chains can be measured over the first 3 weeks of life, indicating an increase in IEC PARP activity. These changes are accompanied by increased intestinal growth and IEC proliferation as assessed by villus height/crypt depth, intestinal length, and Ki67 staining.

CONCLUSION: Understanding how IEC GSH/GSSG redox potential is developmentally regulated may provide insight into how premature human intestinal redox states can be manipulated to optimize intestinal growth and adaptation.

O xidative stress has been implicated in many diseases affecting premature infants, including retinopathy of prematurity, chronic lung disease, intraventricular hemorrhage, periventricular leukomalacia, and necrotizing enterocolitis (NEC) (1). However, clinical studies have failed to demonstrate improved outcomes with antioxidant administration to premature infants $(2-4)$. This is likely because physiologic reactive oxygen species (ROS) signaling regulates many necessary, homeostatic processes, which are negatively affected by global ROS suppression through massive antioxidant supplementation approaches (5). Intracellular ROS signaling and redox potential has been implicated in regulating developmental processes in the fetus and premature newborn, and depends on tightly regulated changes in cellular localization and concentration (6-8). In particular, the intracellular redox potential $\left(E_{\mathrm{h}}\right)$, as determined by the Nernst equation using glutathione (GSH) and glutathione disulfide (GSSG) concentrations, has a key role in regulating important developmental cellular processes, such as proliferation and differentiation (9-11).

In vitro studies indicate that a reduced GSH/GSSG redox potential favors proliferation, whereas an oxidized redox potential favors differentiation $(10,12)$. Developmental regulation of intestinal growth and adaptation depends upon an appropriate balance between the two. However, how the ontogeny of intestinal epithelial cellular (IEC) GSH/GSSG redox regulates these processes in the developing intestine has not been characterized in vivo. Here, we show that IEC GSH/ GSSG redox potential becomes increasingly reduced (primarily driven by a more than fourfold increase in IEC GSH concentration) over the first 3 weeks of life in all regions of the murine intestine. Consistent with the idea that reduced intracellular GSH/GSSG redox potential increases cellular proliferation, we found an increase in intestinal growth and IEC proliferation as assessed by villus height/crypt depth, intestinal length, and Ki67 staining over the first 3 weeks of murine life.

IEC proliferation in the developing gut is an important host defense mechanism. The premature infant intestine is expected to double in length postnatally to match in utero growth rates (13). Further, IEC proliferation in response to injury is critical to promote wound healing and recovery from disease. This defense mechanism has a key role not only in the postnatal maturation of gut function in the preterm neonate but also in how neonates and children recover from intestinal injury that may be chemically induced (i.e., prostaglandin

\footnotetext{
${ }^{1}$ Division of Neonatal-Perinatal Medicine, Department of Pediatrics, Emory University School of Medicine, Atlanta, Georgia; ${ }^{2}$ Division of Epithelial Pathobiology, Department of Pathology and Laboratory Medicine, Emory University School of Medicine, Atlanta, Georgia; ${ }^{3}$ Department of Physiology and Developmental Biology, College of Life Sciences, Brigham Young University, Provo, Utah. Correspondence: Patricia W. Denning (pllin@emory.edu) 


\section{Ontogeny of IEC GSH redox potential Articles}

inhibitors), infection induced, or surgically induced (for NEC or congenital bowel defects) (14). Thus, understanding the potential mechanisms behind GSH-induced IEC proliferation is important in order to provide insight into how neonatal intestinal redox states can be manipulated to optimize intestinal growth and adaptation.

One mechanism by which increases in intracellular GSH have been shown to drive cellular proliferation is through increased poly-ADP-ribose polymerase (PARP) activity (15). To determine whether the developmental increase in intracellular GSH-induced IEC proliferation is accompanied by increasing PARP activity, we measured the amount of intracellular poly-ADP-ribose chains in the neonatal intestine over the first 3 weeks of life. We found that IEC poly-ADPribose chains increased over the first 3 weeks of life in the immature murine intestine, indicating an increase in IEC PARP activity. These studies indicate that developmentally regulated increases in intracellular GSH concentration may promote IEC proliferation by inducing IEC PARP activity in the developing murine gut. Thus, nutritional or pharmacologic strategies that encourage a more reduced IEC intracellular GSH/GSSG state in the immature intestine may be critical to maintaining intestinal health during development and following intestinal injury in premature infants.

\section{METHODS}

\section{Animal Care}

C57BL/6J mice were bred at an animal facility at Emory University, and all studies were approved by the Institutional Animal Care and Use Committee. Neonatal and adult mice were anesthetized with $\mathrm{CO}_{2}$ and killed by cervical dislocation at the ages indicated. Whole proximal small intestine, distal small intestine, and colon were isolated and fixed in $10 \%$ formalin (for histologic staining) or frozen in Trizol (for qRT-PCR), or freshly collected intestinal epithelial cells were scraped into ice-cold perchloric acid (PCA) solution (for GSH analysis) or ice-cold RIPA lysis buffer (for PAR western blot analysis).

\section{GSH Assay}

To assay the GSH antioxidant pool, we measured both GSH and GSSG concentrations by HPLC as $S$-carboxymethyl, $N$-dansyl derivatives using $\gamma$-glutamyl-gluatmate as an internal standard as previously described $(16,17)$. Intestinal epithelial cell scrapings were immediately placed in cold 5\% PCA buffer containing $10 \mu \mathrm{M}$ $\gamma$-glutamyl-glutamate. Samples were subsequently derivatized, analyzed by HPLC, and intracellular GSH and GSSG concentrations were determined (normalized to protein concentration) and used in the Nernst equation to determine the redox potential (18).

\section{qRT-PCR}

Proximal small intestine, distal small intestine, and colon were isolated from mice of the desired ages into Trizol (Invitrogen, Grand Island, NY). Samples were briefly sonicated and total RNA was isolated and reverse transcribed from random hexamer primers using the QuantiTect Reverse Transcription Kit (Qiagen, Carol Stream, IL). The resulting cDNA products were analyzed by real-time quantitative RT-PCR (iQ SYBR Green Supermix on MyiQ real-time PCR detection system, Biorad, Hercules, CA) for GCLC and 18s ribosomal RNA. The level of GCLC expression was normalized to the $18 \mathrm{~s}$ rRNA of the same sample. Fold difference was the ratio of the normalized value of each sample compared with the average 2-day results.

18s forward primers: GGCGCCGGCGGCTTGGTGACTCTA

18s reverse primers: CGCGCCTGCTGCCTTCCTTGGATGT

\section{GCLC forward primers: AGCATCTGGAGAACTAATGACTG} GCLC reverse primers: CAAGTAACTCTGGACATTCACAC Primer source: Ungvari et al. (19).

\section{Histologic Staining}

Proximal small intestine, distal small intestine, and colon were isolated from mice of the desired ages and fixed in $10 \%$ formalin. Samples were paraffin embedded and cut into $5 \mu \mathrm{m}$ sections. Standard hematoxylin and eosin staining was done, and images were taken using a Nikon Eclipse 50i microscope (Melville, NY, USA). Slides stained for Ki67 were deparaffinized in xylene, then treated with $0.9 \%$ hydrogen peroxide in methanol, and rehydrated. Antigen retrieval was performed in a $10 \mathrm{~mm}$ citrate buffer $(\mathrm{pH}$ 6) in a pressure cooker. The slides were blocked using $2 \%$ dry milk in PBST. They were probed with anti-Ki-67 (Abcam, Cambridge, MA), followed by biotin-conjugated anti-rabbit $\operatorname{IgG}(\mathrm{H}+\mathrm{L})$ (Pierce Antibodies, Rockford, IL) and streptavidin-HRP (Abcam). The slides were then developed using DAB Substrate Kit (Invitrogen, Grand Island, NY) and counterstained with hematoxylin (Invitrogen). Images were taken using a Nikon Eclipse 50i microscope and Ki-67positive cells were quantified.

\section{PAR ELISA}

For ELISA analysis of PAR, murine intestinal epithelial cells were scraped into ice-cold RIPA lysis buffer containing protease inhibitors (Roche Applied Science, Indianapolis, IN), sonicated, and centrifuged to remove debris. Protein concentration was assayed by DC Protein Assay Kit II (BioRad, Hercules, CA) and measured with a SpectraMax Plus384 (Molecular Devices, Sunnyvale, CA). PAR was detected using PAR ELISA kit "PARP in vivo Pharmacodynamic Assay 2nd Generation (PDA II)" according to the manufacturer's instructions (Trevigen, Gaithersburg, MD). Luminescence was measured using a FLUOstar OPTIMA (BMG Labtech, Ortenburg, Germany). Samples were all run in triplicate. PAR concentrations were normalized to protein concentration for each sample.

\section{BSO Treatment}

Two-week-old animals were given $0.22 \mathrm{mg}$ BSO or an equivalent volume of sterile phosphate-buffered saline by oral gavage daily for 1 week. The animals were subsequently killed $4 \mathrm{~h}$ after the final administration. The small intestine and colon were harvested into $10 \%$ formalin for histological analysis, or intestinal epithelial cell scrapings were collected into ice-cold 5\% PCA buffer containing $10 \mu \mathrm{M} \gamma$-glutamyl-glutamate for GSH assay by HPLC analysis as described above.

\section{Statistical Analysis}

For comparison among multiple groups, data were first tested for normality using the Kolmogorov-Smirnov test. If normality was established, statistical differences were determined by parametric one-way analysis of variance (Dunnett's multiple comparison posttest). If normality was not established or $N$ was determined to be too small for normality test, statistical differences were determined by the Kruskal-Wallis and Dunn's test (non-parametric test) and Dunns multiple comparison test. For comparison between two groups, a Student's $t$-test was performed. A $P<0.05$ was considered statistically significant.

\section{RESULTS}

\section{Intestinal Epithelial Cell GSH/GSSG Redox Potential Reduces} Over the First 3 Weeks of Postnatal Murine Life

To characterize the ontogeny of IEC GSH/GSSG redox potential, we isolated intestinal epithelia from the proximal small intestine, distal small intestine, and colon of neonatal mice over the first 3 weeks of life, and we measured GSH and GSSG concentrations by high-performance liquid chromatography (HPLC). The IEC 

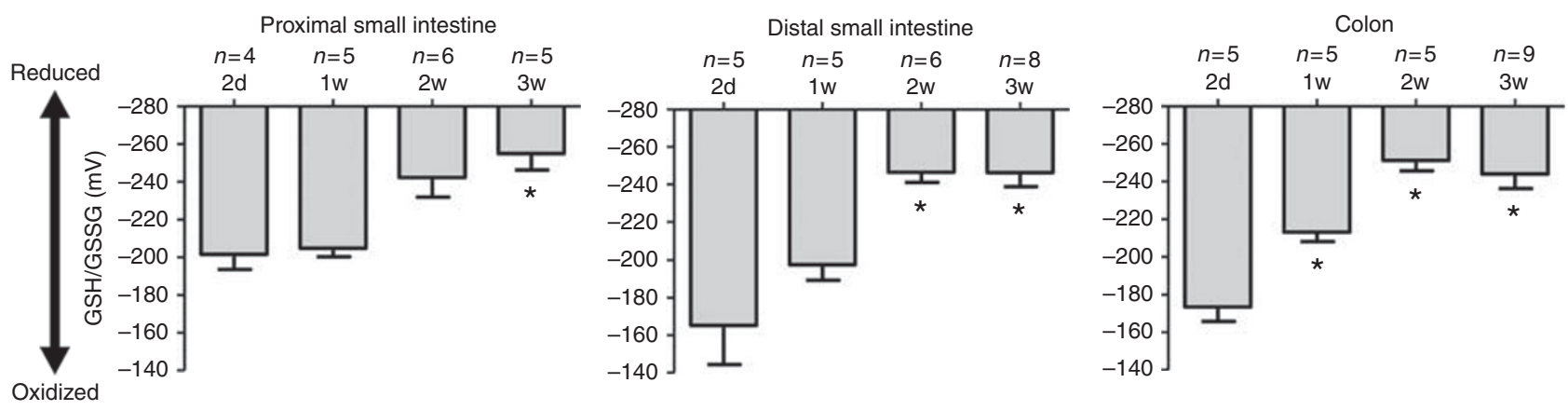

Figure 1. IEC glutathione (GSH)/glutathione disulfide (GSSG) redox potential reduces over the first 3 weeks of postnatal murine life. GSH/GSSG redox potential in epithelial cells isolated from different regions of the murine intestine at various postnatal ages. Data are means \pm s.e.m. ${ }^{*} P<0.05$ when compared with 2 day condition.

GSH/GSSG redox potential became increasingly reduced over the first 3 weeks of life in all regions of the murine intestine (from $-202 \mathrm{mV}$ at 2 days to $-265 \mathrm{mV}$ at 3 weeks in the proximal small intestine; from $-165 \mathrm{mV}$ at 2 days to $-246 \mathrm{mV}$ at 3 weeks in the distal small intestine; and from $-174 \mathrm{mV}$ at 2 days to $-244 \mathrm{mV}$ at 3 weeks in the colon, Figure 1). This change in IEC GSH/GSSG redox potential was driven primarily by an increase of more than ninefold in IEC GSH concentration ( $1.5 \mathrm{~mm}$ at 2 days to $18.3 \mathrm{~mm}$ at 3 weeks in the proximal small intestine; $1.5 \mathrm{~mm}$ at 2 days to $13.5 \mathrm{~mm}$ at 3 weeks in the distal small intestine; and $1.1 \mathrm{~mm}$ at 2 days to $12.6 \mathrm{~mm}$ at 3 weeks in the colon) (Figure 2a). This change was accompanied by an increase in gamma-glutamylcysteinyl synthetase catalytic subunit $(g c l c$, the catalytic subunit of the rate limiting enzyme for de novo GSH synthesis) mRNA expression (Figure $2 \mathbf{b}$ ). IEC GSSG concentration remained stable over the first 3 weeks of life (Figure 2c).

Intestinal Epithelial Cell Proliferation Increases Over the First 3 Weeks of Postnatal Murine Life Resulting in Increased Villus Height, Crypt Depth, and Intestinal Length

As previous in vitro studies have indicated that a reduced GSH/GSSG redox potential favors cellular proliferation, we investigated the ontogeny of IEC proliferation rates by measuring Ki67-positive cells in the proximal small intestine, distal small intestine, and colon of neonatal mice over the first 3 weeks of life. As expected, IEC proliferation, as measured by positive Ki67 staining, increased in all regions of the intestine over the first 3 weeks of life (from 10 to 20 positive cells per crypt from 2 days to 3 weeks in the proximal small intestine; from 11 to 14 positive cells per crypt from 2 days to 3 weeks in the distal small intestine; and from 7 to 11 positive cells per crypt from 2 days to 3 weeks in the colon, Figure 3). This was accompanied by an increase in villus height, crypt depth (from 164 to $295 \mu \mathrm{m}$ from 2 days to 3 weeks in the proximal small intestine; from 112 to $179 \mu \mathrm{m}$ from 2 days to 3 weeks in the distal small intestine; and from 95 to $250 \mu \mathrm{m}$ from 2 days to 3 weeks in the colon, Figure 4a), and intestinal length in both the small and large intestines (from 8 to $20 \mathrm{~cm}$ from 2 days to 3 weeks in the small intestine and from 2 to $4 \mathrm{~cm}$ from 2 days to 3 weeks in the colon, Figure $4 \mathbf{b}$ ).
Intestinal Epithelial Cell Intracellular Concentration of Poly-ADPRibose Chains Increases Over the First 3 Weeks of Postnatal Murine Life, Indicating Increased PARP Activity

Increases in intracellular GSH have been shown to drive cellular proliferation through increased PARP activity (15). To determine whether increased IEC proliferation in the developing neonatal mouse could also be driven by a similar mechanism, we assessed PARP activity by measuring intracellular epithelial PAR chains in the proximal small intestine, distal small intestine, and colon by means of enzyme-linked immunosorbent assay (ELISA). Increasing intracellular epithelial PAR chains were detectable in all regions of the intestine over the first 3 weeks of life, indicating increasing PARP activity (from 5 to $919 \mathrm{pg} / \mathrm{g}$ from 2 days to 3 weeks in the proximal small intestine; from 231 to $1,279 \mathrm{pg} / \mathrm{g}$ from 2 days to 3 weeks in the distal small intestine; and from 812 to $1,855 \mathrm{pg} / \mathrm{g}$ from 2 days to 3 weeks in the colon, Figure 5).

Inhibition of GSH Synthesis Prevents Developmental Reduction in Glutathione Redox Potential, Prevents Developmental Increase in IEC Glutathione, and Reduces IEC Proliferation in the Neonatal Murine Colon

Finally, we sought to determine whether some of the developmental changes observed over the first 3 weeks of neonatal murine life can be inhibited by inhibiting GSH synthesis. To do so, we treated 2-week-old mice with carrier control or L-buthionine-sulfoximine (BSO), an inhibitor of gamma-glutamylcysteinyl synthetase (the rate limiting enzyme of GSH synthesis), daily by means of oral gavage for 1 week. We subsequently measured IEC glutathione redox potential, IEC GSH concentration, and IEC proliferation in the small intestine and colon as described above. Unexpectedly, BSO treatment had no effect on IEC glutathione redox potential in the small intestine, whereas it did prevent developmental reduction in IEC glutathione redox potential in the colon (Figure 6a). Similarly, BSO treatment had no effect on IEC GSH concentration, whereas it did block the developmental increase in IEC GSH concentration in the colon (Figure 6b). We speculate that the more proximal portions of the intestine (small intestine) were able to use dietary intake of GSH to regulate GSH concentrations and 
a

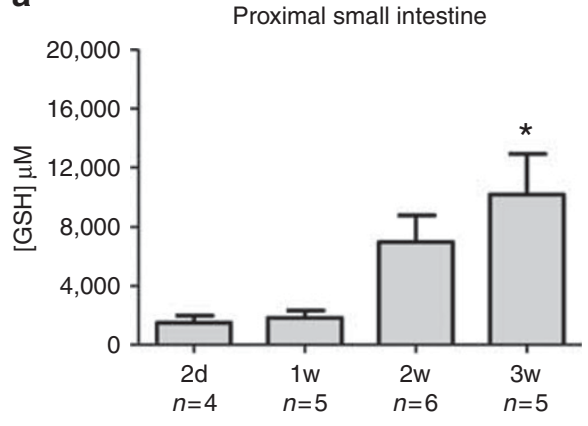

b

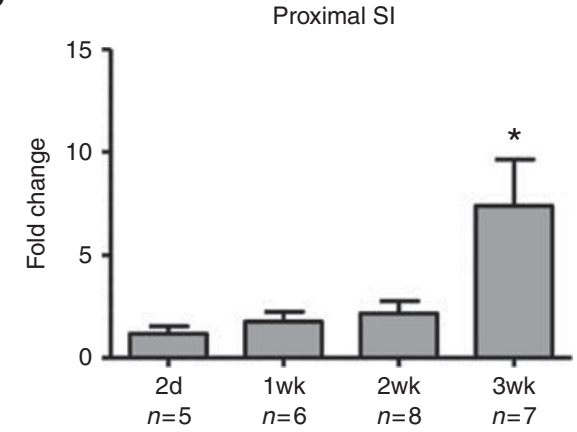

C

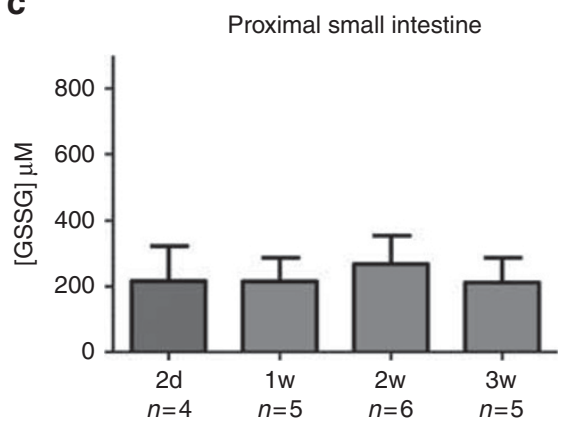

Distal small intestine

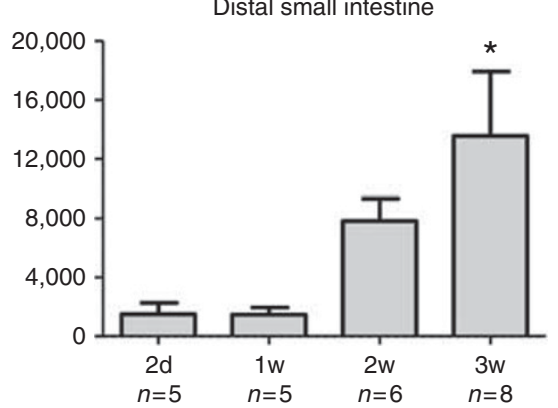

Distal SI

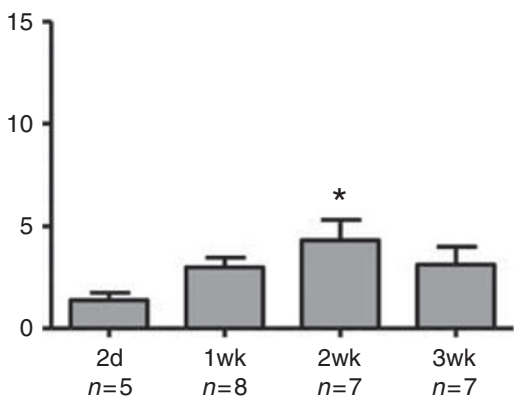

Distal small intestine

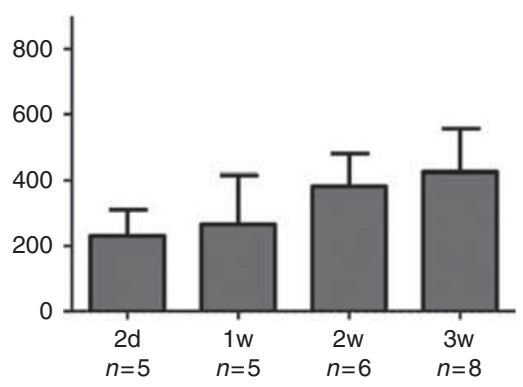

Colon
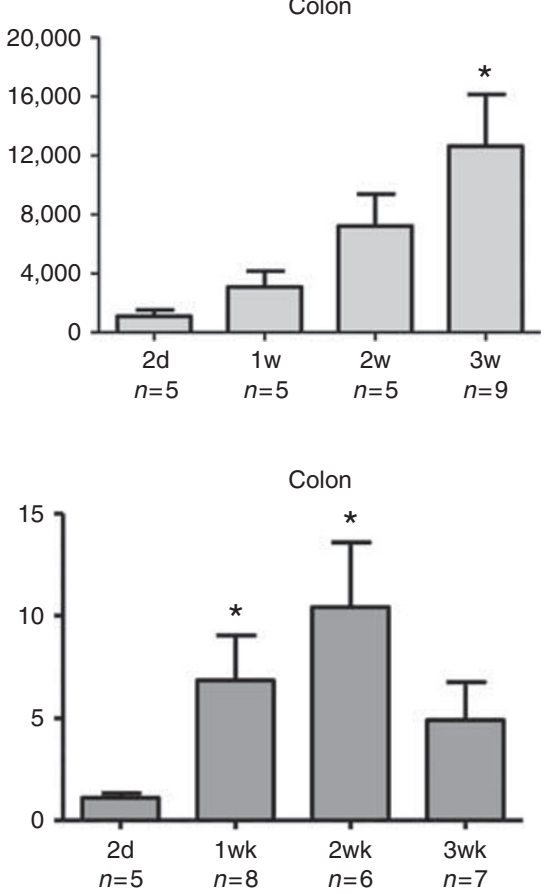

Colon

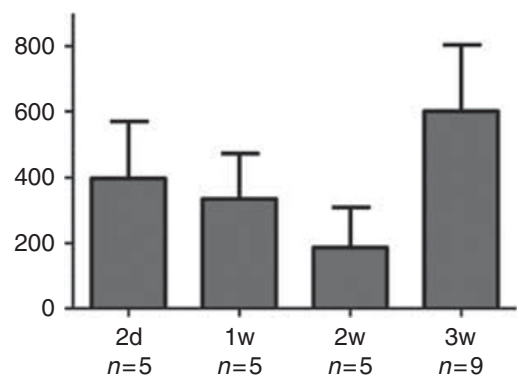

Figure 2. Intestinal epithelial cellular (IEC) [GSH] increases, whereas [GSSG] remains stable over the first 3 weeks of postnatal murine life. (a) Glutathione concentration $[\mathrm{GSH}]$ in epithelial cells isolated from different regions of the murine intestine at various postnatal ages. (b) Fold change in gclc (gamma-glutamylcysteinyl synthetase catalytic subunit of the rate limiting enzyme for de novo glutathione (GSH) synthesis) mRNA expression in epithelial cells isolated from different regions of the murine intestine at various postnatal ages as assessed by qRT-PCR. (c) Glutathione disulfide concentration [GSSG] in epithelial cells isolated from different regions of the murine intestine at various postnatal ages. Data are means \pm s.e.m. ${ }^{*} P<0.05$ when compared with 2 day condition.
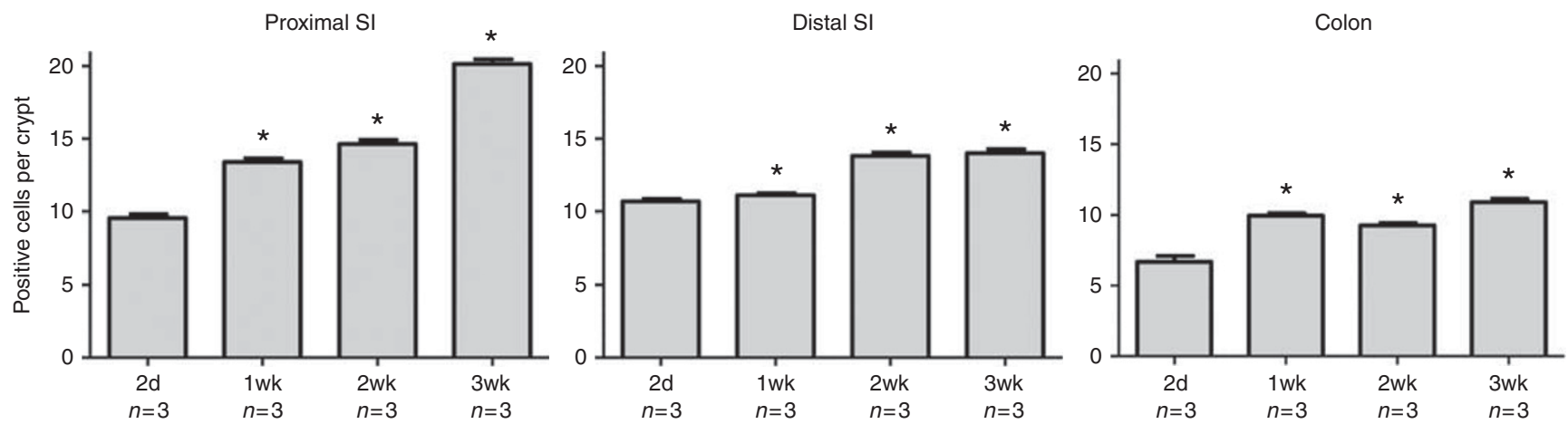

Figure 3. Intestinal epithelial cellular (IEC) proliferation as assessed by Ki67 staining increases over the first 3 weeks of postnatal murine life. Number of Ki67-positive cells per crypt in different regions of the murine intestine over the first 3 weeks of postnatal life. Data are means \pm s.e.m. ${ }^{*} P<0.05$ when compared with 2 day condition. 
a
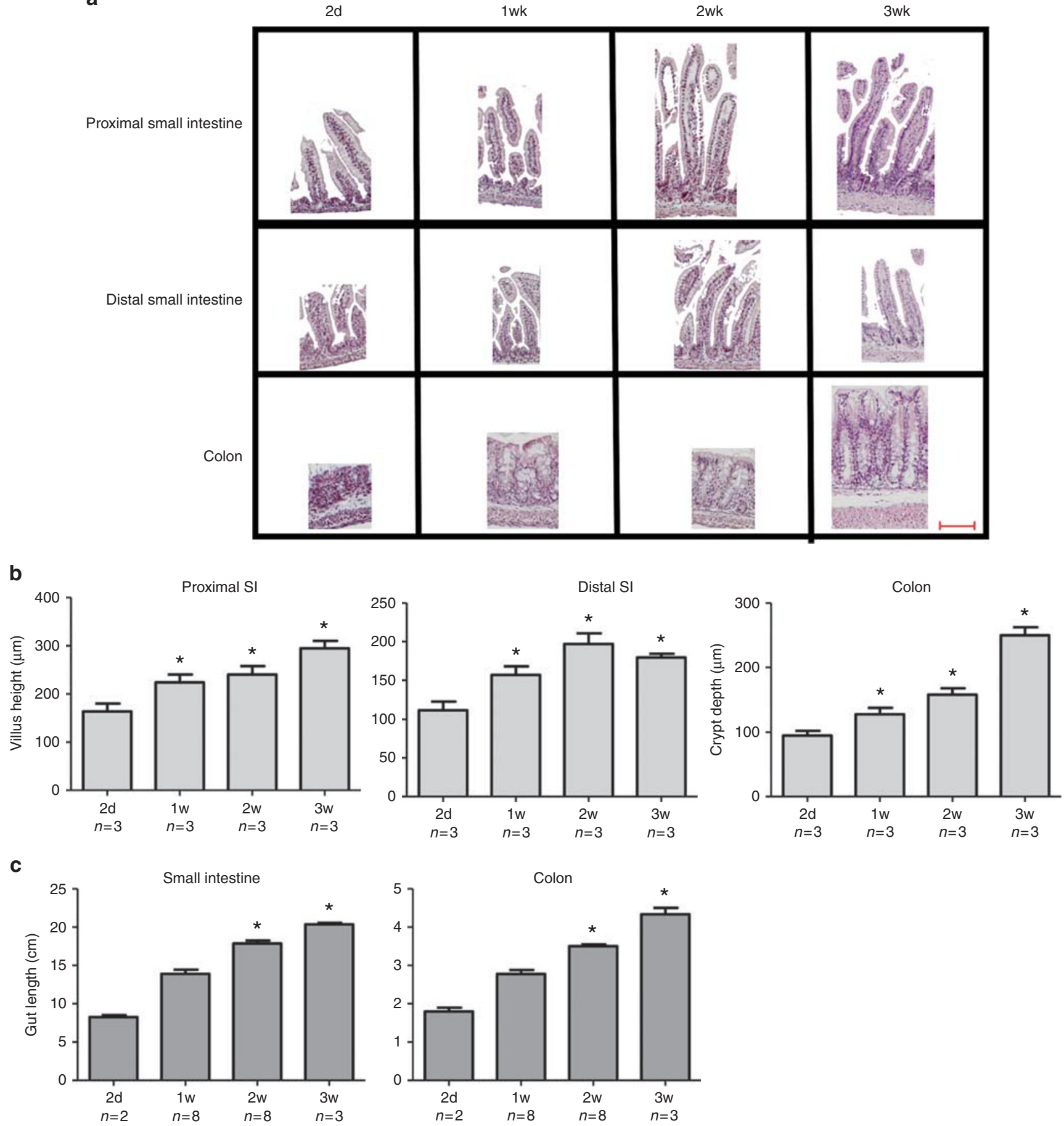

Figure 4. Intestinal villus height, crypt depth, and intestinal length increases over the first 3 weeks of postnatal murine life. (a) H\&E staining of murine intestine over the first 3 weeks of postnatal life. Scale bar represents $100 \mu \mathrm{m}$. (b) Villus height/crypt depth (mean \pm s.e.m.) in different regions of the murine intestine over the first 3 weeks of postnatal life. (c) Intestinal length of small intestine and colon (mean \pm s.e.m.) over the first 3 weeks of postnatal life. ${ }^{*} P<0.05$ when compared with 2 day condition.

compensate for the lack of GSH synthesis. Finally, we demonstrated a reduction in Ki67-positive cells in the colon of neonatal mice treated with BSO for a week, whereas we found no reduction in Ki67-positive cells in the small intestine of neonatal mice treated with BSO for a week
(Figure 6c). These results indicate that $\mathrm{BSO}$ can reduce intracellular GSH and IEC proliferation in the developing colon and are consistent with our idea that increased intracellular GSH may drive increased IEC proliferation developmentally in the neonatal murine intestine. 

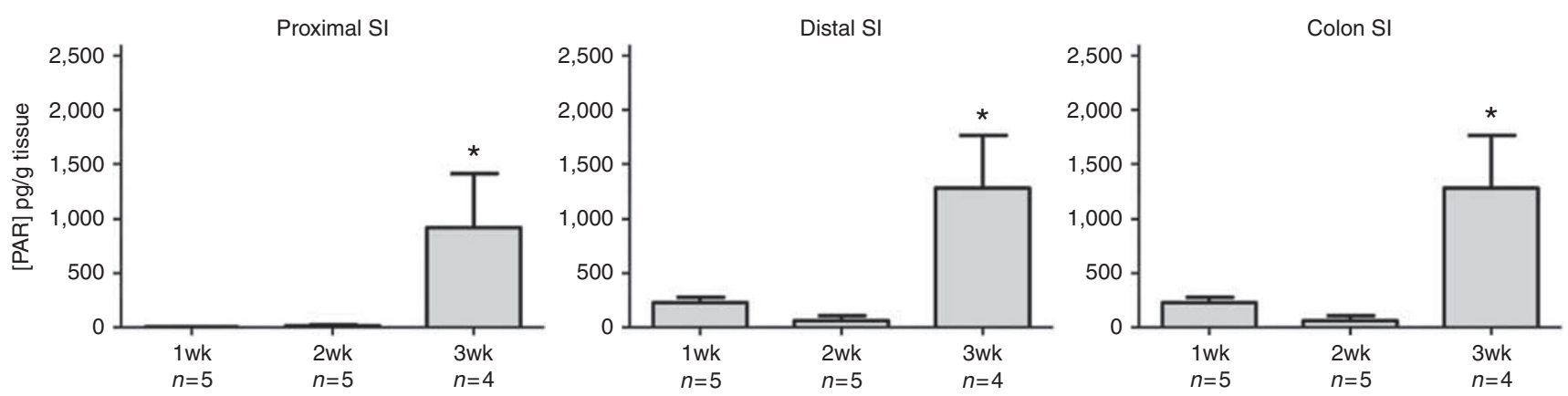

Figure 5. Intestinal epithelial cellular (IEC) poly-ADP-ribose polymerase (PARP) activity increases over the first 3 weeks of murine life. Concentration of intracellular epithelial PAR chains as assessed by ELISA from IEC isolated from different regions of the murine intestine at various postnatal ages (normalized to protein concentration of each sample). Data are means \pm s.e.m. ${ }^{*} P<0.05$ when compared with 1 week condition.
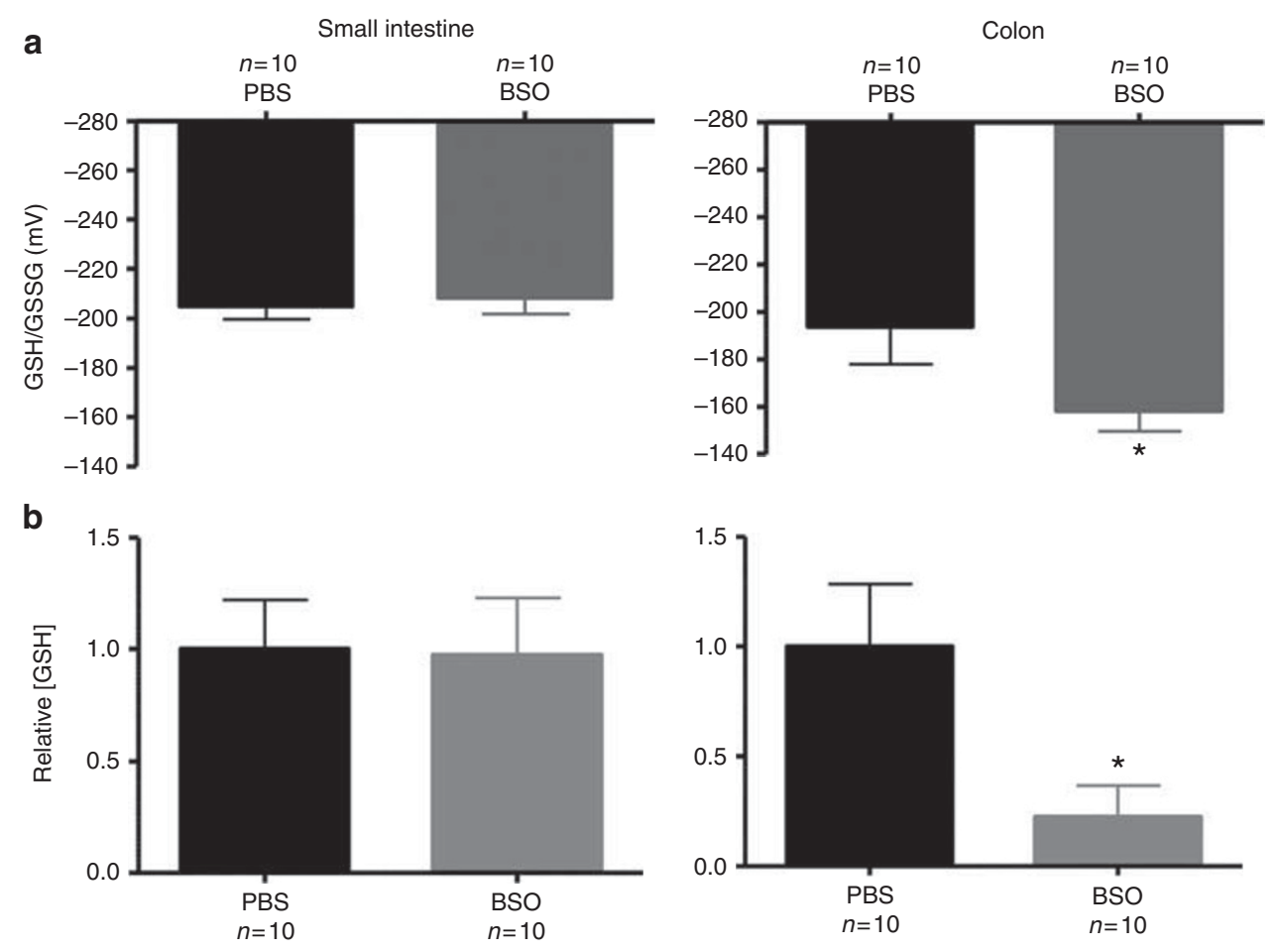

C
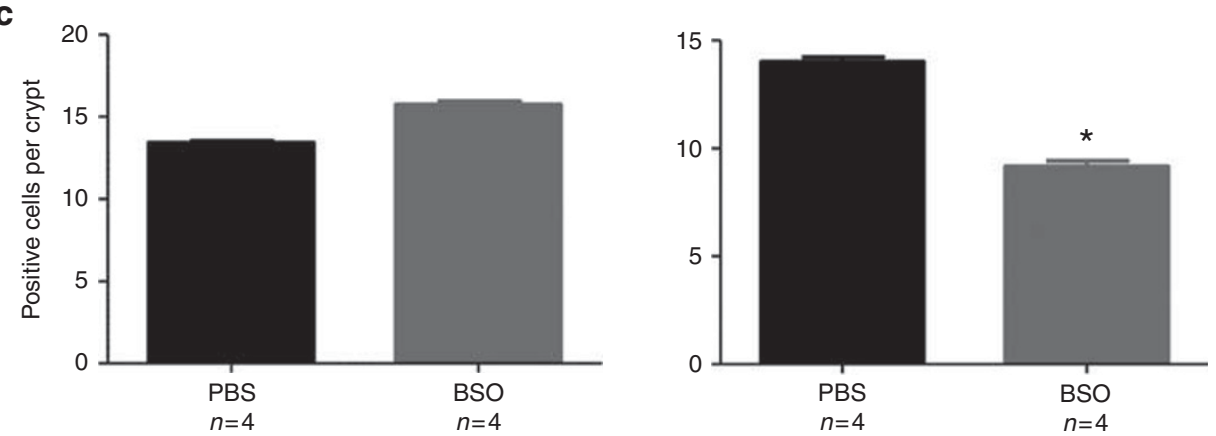

Figure 6. Reduction in intestinal epithelial cellular (IEC) glutathione reduces IEC proliferation in neonatal mice. (a) Glutathione (GSH)/glutathione disulfide (GSSG) redox potential in 3-week-old neonatal mice treated with phosphate-buffered saline \pm L-buthionine-sulfoximine (BSO) once daily for a week. (b) Relative IEC glutathione concentration in 3-week-old neonatal mice treated with phosphate-buffered saline \pm BSO for a week. (c) Number of Ki67-positive cells per crypt in 3-week-old neonatal mice treated with phosphate-buffered saline \pm BSO for a week. Data are means \pm s.e.m. ${ }^{*} P<0.05$. 


\section{Articles | Reid et al.}

\section{DISCUSSION}

The immature intestine of the premature infant may be at greater risk for intestinal injury and inflammation (antecedents of NEC) due to defects in barrier function, innate immunity, motility, digestion, and circulatory regulation (20,21). Thus, developing strategies to promote maturation of the premature gut have become a stated priority (22). In particular, the intracellular redox potential of GSH/GSSG is known to have a key role in regulating proliferation and differentiation (9). In an effort to determine how intracellular redox states in the neonatal intestine may be targeted to promote intestinal homeostasis in the premature infant, this study aimed to characterize the ontogeny of intestinal epithelial intracellular GSH/GSSG redox potential using a murine model of immature intestine.

Using this model, we demonstrated that the IEC GSH/ GSSG redox potential becomes increasingly reduced during postnatal intestinal maturation. This change was primarily driven by an increase in intracellular GSH concentration in the second week of murine life. Developmentally, the 2-weekold murine intestine is thought to correspond to a 24 -weekold premature human intestine (23). Thus, significant increases in IEC GSH production may influence appropriate development of premature human intestine at this time. Disruptions to this process may negatively affect important developmental processes and potentially reduce the infant's ability to respond to such injury. As the premature intestine adapts to extrauterine life, it is at risk for injury from multiple sources, such as microbes, nutrients, and medications. As reduced intracellular GSH/GSSG may be important to drive IEC proliferation, disruption in the maturation of the IEC GSH/GSSG redox potential may hinder the ability of the immature intestine to respond to epithelial injury with proliferation. Epithelial injury may further reduce intestinal barrier function, leading to invasion with toxins or microbes causing inflammation and additional injury, resulting in a vicious cycle that could have serious consequences (21).

The premature infant is at particularly high risk for developing diseases related to oxidative stress because the preterm infant has prematurely transitioned from the hypoxic in utero environment (in utero) to the relatively hyperoxic extrauterine environment. Furthermore, premature infants have underdeveloped antioxidant systems to counteract exogenous and endogenous sources of oxidative stress (24-26). Our study indicates that maturation of the GSH antioxidant system may be important for maturation and growth of the neonatal intestine. This may be occurring in utero in the fetus and postnatally in the premature neonate. Thus, oxidative stress in the preterm neonate may negatively affect continued postnatal maturation and growth of the immature intestine. In vitro studies indicate that highly reduced GSH redox states $(-250 \mathrm{mV})$ favor cellular proliferation, slightly oxidized GSH redox states $(-220 \mathrm{mV})$ favor cellular differentiation, whereas further oxidation promotes cellular apoptosis or necrosis $(<-190 \mathrm{mV})(27,28)$. In our study, IEC GSH redox potentials seen at 2-3 weeks in the neonatal murine intestine were consistent with an environment promoting cellular proliferation
( -240 to $-265 \mathrm{mV}$ range), whereas the IEC GSH redox potential seen at 1 week was consistent with an environment promoting cellular differentiation ( -200 to $-215 \mathrm{mV}$ range). In the more distal portions of the intestine (distal SI and colon), 2day-old murine IEC exhibited GSH redox potentials consistent with an environment promoting apoptosis or necrosis ( -165 to $-175 \mathrm{mV}$ range). Thus, preterm neonates not able to induce more robust IEC GSH synthesis may exhibit prolonged periods of more oxidized IEC redox potentials $(<-190 \mathrm{mV})$ and may potentially be predisposed to IEC apoptosis or necrosis. This may ultimately result in preterm intestinal diseases such as spontaneous intestinal perforation (SIP) and NEC. Consistent with this idea, hyperoxia causes NEC-like injury in neonatal animals (29); animal and human studies suggest that apoptosis may be an early pathogenic factor in NEC development (30-32); and distal SI and colon are commonly affected in both SIP and NEC (33-35). Further, hyperoxic injury has been used as an animal model of NEC (29).

Inflammatory processes are also known to lead to increased ROS production that may further influence GSH/GSSG redox imbalance in the IEC, causing impaired healing in response to injury. Future studies investigating how to restore this balance in the preterm intestine may lead to better preventive and therapeutic strategies for neonatal intestinal diseases such as SIP and NEC. As ROS are known to function as messenger molecules that serve to regulate homeostatic cellular functions, global normalization of ROS production via antioxidant therapies will likely not be an ideal strategy. Indeed, clinical trials of antioxidant therapy in preterm infants have largely failed to yield improved outcomes (2-4). In this study, changes in reduction of GSH redox potentials were primarily driven by the increased GSH concentrations. Studies in adult mice confirm that blocking GSH production causes intestinal epithelial cell damage (36). Enteral GSH administration can restore intestinal GSH levels and reverse epithelial damage $(37,38)$. Further, dietary restriction of sulfur amino acid intake has also been shown to negatively influence intestinal GSH levels (39), whereas sulfur amino acid supplementation has been shown to improve intestinal adaptation after small bowel resection in vivo via effects on intestinal mucosal redox potential (40). Kertinocyte growth factor has also been shown to improve intestinal mucosal growth by increasing its GSH content (41). This suggests that nutritional or pharmacologic strategies to optimize GSH levels or production may be a viable strategy to normalize GSH redox potential to promote homeostasis in the preterm intestine without suppressing physiologic ROS function.

\section{ACKNOWLEDGMENTS}

We would like to acknowledge Loren S. Myers for her technical support toward completion of the studies reported.

Disclosure: The authors declare no conflict of interest.

\section{REFERENCES}

1. Marseglia L, D'Angelo G, Manti S, et al. Oxidative stress-mediated aging during the fetal and perinatal periods. Oxid Med Cell Longev 2014;2014: 358375 . 


\section{Ontogeny of IEC GSH redox potential $\mid$ Articles}

2. Brion LP, Bell EF, Raghuveer TS. Vitamin E supplementation for prevention of morbidity and mortality in preterm infants. Cochrane Database Syst Rev 2003;3:CD003665.

3. Darlow BA, Austin NC. Selenium supplementation to prevent short-term morbidity in preterm neonates. Cochrane Database Syst Rev 2003;4: CD003312.

4. Suresh GK, Davis JM, Soll RF. Superoxide dismutase for preventing chronic lung disease in mechanically ventilated preterm infants. Cochrane Database Syst Rev 2001;1:CD001968.

5. Veal EA, Day AM, Morgan BA. Hydrogen peroxide sensing and signaling. Mol Cell 2007;26:1-14.

6. Covarrubias L, Hernandez-Garcia D, Schnabel D, Salas-Vidal E, Castro-Obregon S. Function of reactive oxygen species during animal development: passive or active? Dev Biol 2008;320:1-11.

7. Das KC. Redox control of premature birth and newborn biology. Antioxid Redox Signal 2004;6:105-7.

8. Lavoie JC, Chessex P. Development of glutathione synthesis and gammaglutamyltranspeptidase activities in tissues from newborn infants. Free Radic Biol Med 1998;24:994-1001.

9. Hernandez-Garcia D, Wood CD, Castro-Obregon S, Covarrubias L. Reactive oxygen species: a radical role in development? Free Radic Biol Med 2010;49:130-43.

10. Hutter DE, Till BG, Greene JJ. Redox state changes in density-dependent regulation of proliferation. Exp Cell Res 1997;232:435-8.

11. Imhoff BR, Hansen JM. Differential redox potential profiles during adipogenesis and osteogenesis. Cell Mol Biol Lett 2011;16:149-61.

12. Nkabyo YS, Ziegler TR, Gu LH, Watson WH, Jones DP. Glutathione and thioredoxin redox during differentiation in human colon epithelial (Caco2) cells. Am J Physiol Gastrointest Liver Physiol 2002;283:G1352-9.

13. Touloukian RJ, Smith GJ. Normal intestinal length in preterm infants. J Pediatr Surg 1983;18:720-3.

14. Kelly N, Friend K, Boyle P, et al. The role of the glutathione antioxidant system in gut barrier failure in a rodent model of experimental necrotizing enterocolitis. Surgery 2004;136:557-66.

15. Pellny TK, Locato V, Vivancos PD, et al. Pyridine nucleotide cycling and control of intracellular redox state in relation to poly (ADP-ribose) polymerase activity and nuclear localization of glutathione during exponential growth of Arabidopsis cells in culture. Mol Plant 2009;2:442-56.

16. Jones DP. Redox potential of GSH/GSSG couple: assay and biological significance. Methods Enzymol 2002;348:93-112.

17. Lin PW, Myers LE, Ray L, et al. Lactobacillus rhamnosus blocks inflammatory signaling in vivo via reactive oxygen species generation. Free Radic Biol Med 2009;47:1205-11.

18. Hansen JM, Harris C. Glutathione during embryonic development. Biochim Biophys Acta 2014;1850:1527-42.

19. Ungvari Z, Bailey-Downs L, Gautam T, et al. Adaptive induction of NF-E2related factor-2-driven antioxidant genes in endothelial cells in response to hyperglycemia. Am J Physiol Heart Circ Physiol 2011;300:H1133-40.

20. Neu J, Walker WA. Necrotizing enterocolitis. N Engl J Med 2011;364: 255-64.

21. Lin PW, Stoll BJ. Necrotising enterocolitis. Lancet 2006;368:1271-83.

22. Grave GD, Nelson SA, Walker WA, et al. New therapies and preventive approaches for necrotizing enterocolitis: report of a research planning workshop. Pediatr Res 2007;62:510-14.
23. McCracken VJ, Lorenz RG. The gastrointestinal ecosystem: a precarious alliance among epithelium, immunity and microbiota. Cell Microbiol 2001;3:1-11.

24. Lee JW, Davis JM. Future applications of antioxidants in premature infants. Curr Opin Pediatr 2011;23:161-6.

25. Qanungo S, Mukherjea M. Ontogenic profile of some antioxidants and lipid peroxidation in human placental and fetal tissues. Mol Cell Biochem 2000;215:11-9.

26. Perrone S, Tataranno ML, Negro S, et al. Early identification of the risk for free radical-related diseases in preterm newborns. Early Hum Dev 2010;86:241-4.

27. Schafer FQ, Buettner GR. Redox environment of the cell as viewed through the redox state of the glutathione disulfide/glutathione couple. Free Radic Biol Med 2001;30:1191-212.

28. Denning TL, Takaishi H, Crowe SE, Boldogh I, Jevnikar A, Ernst PB. Oxidative stress induces the expression of Fas and Fas ligand and apoptosis in murine intestinal epithelial cells. Free Radic Biol Med 2002;33:1641-50.

29. Li N, Ma L, Liu X, et al. Arginyl-glutamine dipeptide or docosahexaenoic acid attenuates hyperoxia-induced small intestinal injury in neonatal mice. J Pediatr Gastroenterol Nutr 2012;54:499-504.

30. Ford H, Watkins S, Reblock K, Rowe M. The role of inflammatory cytokines and nitric oxide in the pathogenesis of necrotizing enterocolitis. J Pediatr Surg 1997;32:275-82.

31. Santulli TV, Schullinger JN, Heird WC, et al. Acute necrotizing enterocolitis in infancy: a review of 64 cases. Pediatrics 1975;55: 376-87.

32. Jilling T, Lu J, Jackson M, Caplan MS. Intestinal epithelial apoptosis initiates gross bowel necrosis in an experimental rat model of neonatal necrotizing enterocolitis. Pediatr Res 2004;55:622-9.

33. Bell RS, Graham CB, Stevenson JK. Roentgenologic and clinical manifestations of neonatal necrotizing enterocolitis. Experience with 43 cases. Am J Roentgenol Radium Ther Nucl Med 1971;112:123-34.

34. Gordon PV. Understanding intestinal vulnerability to perforation in the extremely low birth weight infant. Pediatr Res 2009;65:138-44.

35. Tatekawa Y, Muraji T, Imai Y, Nishijima E, Tsugawa C. The mechanism of focal intestinal perforations in neonates with low birth weight. Pediatr Surg Int 1999;15:549-52.

36. Martensson J, Jain A, Meister A. Glutathione is required for intestinal function. Proc Natl Acad Sci USA 1990;87:1715-9.

37. Hagen TM, Wierzbicka GT, Bowman BB, Aw TY, Jones DP. Fate of dietary glutathione: disposition in the gastrointestinal tract. Am J Physiol 1990;259:G530-5.

38. Aw TY, Wierzbicka G, Jones DP. Oral glutathione increases tissue glutathione in vivo. Chem Biol Interact 1991;80:89-97.

39. Nkabyo YS, Gu LH, Jones DP, Ziegler TR. Thiol/disulfide redox status is oxidized in plasma and small intestinal and colonic mucosa of rats with inadequate sulfur amino acid intake. J Nutr 2006;136:1242-8.

40. Shyntum Y, Iyer SS, Tian J, et al. Dietary sulfur amino acid supplementation reduces small bowel thiol/disulfide redox state and stimulates ileal mucosal growth after massive small bowel resection in rats. J Nutr 2009;139:2272-8.

41. Jonas CR, Gu LH, Nkabyo YS, et al. Glutamine and KGF each regulate extracellular thiol/disulfide redox and enhance proliferation in Caco2 cells. Am J Physiol Regul Integr Comp Physiol 2003;285:R1421-9. 